\title{
Safe long-term repeated disruption of the blood-brain barrier using an implantable ultrasound device: a multiparametric study in a primate model
}

\author{
${ }^{*}$ Catherine Horodyckid, MD, ${ }^{1,2}$ Michael Canney, PhD, ${ }^{1}$ Alexandre Vignot, MS, ${ }^{1}$ \\ Raphael Boisgard, PhD, ${ }^{3}$ Aurélie Drier, MD, ${ }^{5}$ Gilles Huberfeld, MD, PhD, ${ }^{4,7-9}$ Chantal François, $\mathrm{PhD},{ }^{10}$ \\ Annick Prigent, ${ }^{11}$ Mathieu D. Santin, PhD, ${ }^{12,13}$ Clovis Adam, MD, ${ }^{14}$ Jean-Claude Willer, MD, PhD, ${ }^{4,6}$ \\ Cyril Lafon, $\mathrm{PhD},{ }^{15}$ Jean-Yves Chapelon, $\mathrm{PhD},{ }^{15}$ and Alexandre Carpentier, MD, PhD ${ }^{2,4}$
}

\begin{abstract}
${ }^{1}$ CarThera Research Team, Brain and Spine Institute, Paris; Departments of ${ }^{2}$ Neurosurgery and ${ }^{6}$ Physiologie, Assistance Publique-Hôpitaux de Paris, Pitié-Salpêtrière Hospital, Paris; ${ }^{3}$ CEA, SHFJ, Orsay; ${ }^{4}$ Paris VI Sorbonne University, School of Medicine, Paris; ${ }^{5}$ Réseau d'Imagerie Parisien, Paris; ${ }^{7}$ INSERM U1129 "Infantile Epilepsies and Brain Plasticity," Paris; ${ }^{8}$ Paris Descartes University, PRES Sorbonne Paris Cité; ${ }^{9} \mathrm{CEA}$, Gif sur Yvette; ${ }^{10}$ Sorbonne Universités, UPMC Univ Paris 06, UMRS 1127, ICM, F-75013 Paris; ${ }^{11}$ Inserm U1127, CNRS UMR 7225, Sorbonne Universités, UPMC Univ Paris 06 UMRS 1127, Institut du Cerveau et de la Moelle épinière, ICM, F-75013, Paris; ${ }^{12}$ Centre de Neurolmagerie de Recherche-CENIR, Paris; ${ }^{13}$ Institut du Cerveau et de la Moelle épinière - ICM, Inserm U 1127, CNRS UMR 7225, Sorbonne Universités, UPMC Univ Paris 06 UMRS 1127, Paris; ${ }^{14}$ Assistance Publique-Hôpitaux de Paris, Bicêtre Hospital, Pathology Department, Le Kremlin-Bicêtre, Paris; and ${ }^{15}$ INSERM U1032, LabTau, University of Lyon, France
\end{abstract}

\begin{abstract}
OBJECTIVE The main limitation to the efficacy of chemotherapy for brain tumors is the restricted access to the brain because of the limited permeability of the blood-brain barrier (BBB). Previous animal studies have shown that the application of pulsed ultrasound (US), in combination with the intravenous injection of microbubbles, can temporarily disrupt the BBB to deliver drugs that normally cannot reach brain tissue. Although many previous studies have been performed with external focused US transducers, the device described in the current work emits US energy using an unfocused transducer implanted in the skull thickness. This method avoids distortion of the US energy by the skull bone and allows for simple, repetitive, and broad disruption of the BBB without the need for MRI monitoring. The purpose of the present study was to determine if the BBB can be safely and repeatedly disrupted using such an implantable unfocused US device in a primate model.
\end{abstract}

METHODS An 11.5-mm-diameter, 1-MHz, planar US device was implanted via a bur hole into the skull of 3 primates (2 Papio anubis [olive] baboons and 1 Macaca fascicularis [macaque]) for 4 months. Pulsed US sonications were applied together with the simultaneous intravenous injection of sulfur hexafluoride microbubbles (SonoVue) every 2 weeks to temporarily disrupt the BBB. In each primate, a total of 7 sonications were performed with a 23.2-msec burst length (25,000 cycles) and a $1-\mathrm{Hz}$ pulse repetition frequency at acoustic pressure levels of $0.6-0.8 \mathrm{MPa}$. Potential toxicity induced by repeated BBB opening was analyzed using MRI, PET, electroencephalography (EEG), somatosensory evoked potential (SSEP) monitoring, behavioral scales, and histopathological analysis.

RESULTS The T1-weighted contrast-enhanced MR images acquired after each sonication exhibited a zone of hypersignal underneath the transducer that persisted for more than 4 hours, indicating a broad region of BBB opening in the acoustic field of the implant. Positron emission tomography images with fluorine-18-labeled fluorodeoxyglucose (FDG) did not indicate any changes in the cerebral metabolism of glucose. Neither epileptic signs nor pathological central nerve conduction was observed on EEG and SSEP recordings, respectively. Behavior in all animals remained normal. Histological analysis showed no hemorrhagic processes, no petechia, and extravasation of only a few erythrocytes.

\footnotetext{
ABBREVIATIONS AUC = area under the standard uptake value time activity curve; BBB = blood-brain barrier; EEG $=$ electroencephalography; FDG $=$ fluorine-18-labeled fluorodeoxyglucose; $\mathrm{Gd}$ = gadolinium; ROI = region of interest; SSEP = somatosensory evoked potential; SUV = standard uptake value; US = ultrasound. SUBMITTED August 16, 2015. ACCEPTED March 24, 2016. INCLUDE WHEN CITING Published online June 10, 2016; DOI: 10.3171/2016.3.JNS151635.

* Drs. Horodyckid and Canney contributed equally to this work.
} 
CONCLUSIONS The studies performed confirm that an implantable, 1-MHz US device can be used to repeatedly open the BBB broadly in a large-animal model without inducing any acute, subacute, or chronic lesions.

https://thejns.org/doi/abs/10.3171/2016.3.JNS151635

KEY WORDS blood-brain barrier; ultrasound; brain tumor; primate; magnetic resonance imaging; drug delivery system; ultrasound contrast agent

$\mathrm{T}$ HE main limitation to the efficacy of chemotherapy in the brain is its restricted access due to the bloodbrain barrier (BBB), which limits the passage of the majority of chemotherapeutic drugs, except for some small $(<0.4 \mathrm{kD})$ or hydrophobic molecules, and thus prevents most chemotherapeutics from crossing from the vasculature compartment into the brain parenchyma. ${ }^{1,5}$ The limited penetrability of the BBB prevents these drugs from reaching therapeutic levels in the brain. ${ }^{29}$

Several methods for increasing the penetration of drugs in the brain by either disrupting or bypassing the $\mathrm{BBB}$ have been clinically studied. First, the local concentrations of drugs in the brain parenchyma were increased through intraarterial injection, convection-enhanced delivery, or drug-loaded wafers. ${ }^{10}$ Second, the permeability of the BBB was modified using osmotic solutions or vasoactive compounds. ${ }^{6}$ Lastly, specially formulated drugs were designed to bypass the $\mathrm{BBB}$ through receptor-mediated transport mechanisms. ${ }^{8}$ Although such techniques have shown promising results, the difficulties in repeatedly performing them, the acquired resistances, and the potential complications have limited their widespread use in the clinic.

An alternative method for enhancing drug delivery to the brain is the temporary disruption of the BBB with the use of externally applied focused pulsed ultrasound (US). Since $2001,{ }^{15}$ research studies have demonstrated that the permeability of the BBB can be reliably and temporarily increased in a range of animal models, including mice, ${ }^{24}$ rats, ${ }^{21}$ rabbits,,${ }^{3,413-16}$ and primates. ${ }^{7,18,19}$ Typical US parameters in preclinical studies have included frequencies of $200 \mathrm{kHz}-5 \mathrm{MHz}$, duty cycles of $1 \%-10 \%$, and sonication times of 1-2 minutes. With such parameters and a mechanical index greater than $0.46,,^{20,27}$ the BBB can be safely and reversibly opened for $5-8$ hours. ${ }^{28}$

While externally applied focused US is a promising approach for temporarily opening the $\mathrm{BBB}$, the presence of the skull bone has thus far limited the technique from being tested in the clinic. ${ }^{27}$ The skull bone absorbs and distorts the US field, making it difficult to accurately predict the acoustic pressure being delivered in situ, which is important for safely opening the BBB. While large, external hemispherical US arrays have been developed to overcome this problem and to focus the US field through the skull bone, ${ }^{19}$ such devices require head immobilization, head shaving, CT scanning, and MRI monitoring to correct the US field. Furthermore, such devices may not be suited to routine clinical use for diffuse pathologies, for which patients typically undergo repeated chemotherapy sessions up to several times per month.

To circumvent the skull bone, our group has recently developed an MRI-compatible, implantable US device. A transcutaneous bipolar needle is used to activate the device during treatments. The device can be implanted either at the end of a regular resection or surgical biopsy procedure, or during a unique dedicated surgical procedure under local anesthesia. An initial study performed in rabbits, in which we used a nonimplantable prototype US transducer, demonstrated that the BBB could be safely and temporarily disrupted within a large volume in the brain by using a 1-MHz transducer with acoustic pressure levels of 0.3-0.8 MPa, pulse lengths of $10-35 \mathrm{msec}$, a pulse repetition frequency of $1 \mathrm{~Hz}$, and sonication durations of 60-120 seconds. ${ }^{3}$

In the current study, a biocompatible version of the US device was developed and implanted in 3 primates to study the safety and long-term toxicity of repeated BBB disruption using pulsed US. The BBB was temporarily disrupted every 15 days over the course of 4 months to simulate a typical, although shortened, course of an administered chemotherapy regimen that a patient may experience. The potential toxicity of such repeated disruption of the BBB was evaluated using a range of techniques including MRI, PET, and behavioral and electrophysiological monitoring for the duration of the experiment. At the end of the study, extracted brain tissue samples were histologically analyzed to further investigate any potential adverse effects of the treatments.

\section{Methods}

Three primates were used in the study. A 1-MHz US device, shown in Fig. 1, was implanted in each primate and used to disrupt the BBB every 15 days over the course of 4 months. Imaging and testing with MRI, fluorine-18labeled fluorodeoxyglucose (FDG)-PET, electroencephalography (EEG), somatosensory evoked potential (SSEP), and histological analysis were performed according to the experimental plan shown in Fig. 2 to determine if any adverse effects were induced after repeated BBB disruption by US. Additional details on each of the analyses performed are described below.



FIG. 1. Photograph of the implantable US device with the transdermal needle connector used to temporarily disrupt the BBB. 


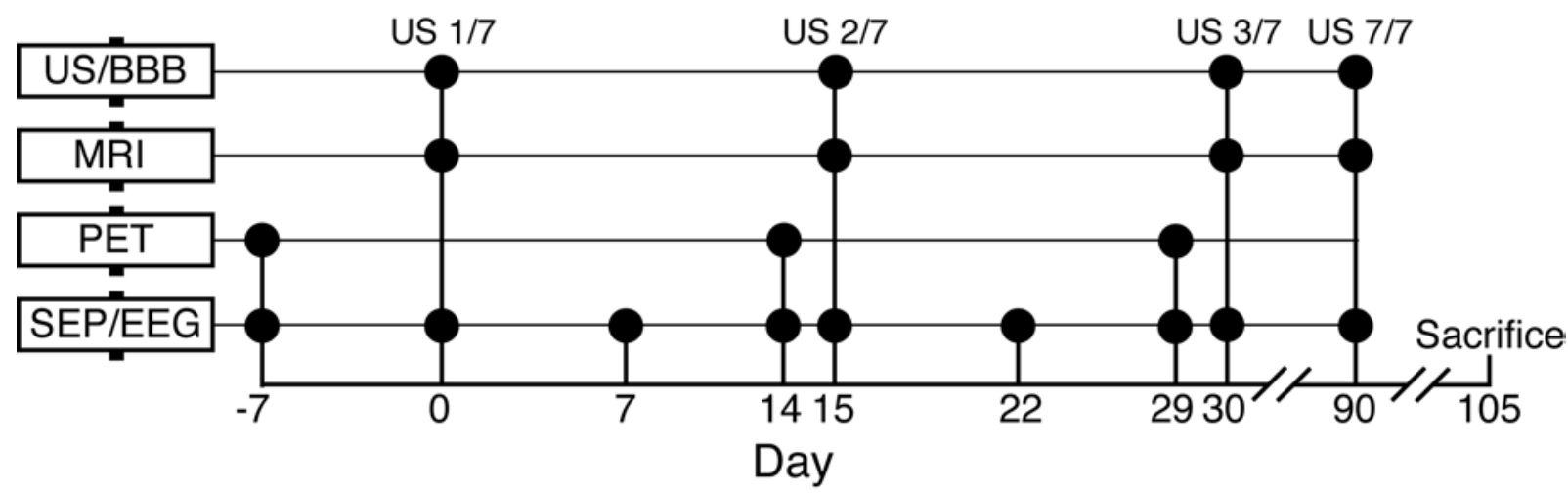

FIG. 2. Diagram of the experimental protocol. Ultrasound (US) was applied approximately every 15 days to disrupt the BBB. Disruption was induced a total of 7 times before each animal was sacrificed and was confirmed using MRI. Between each BBB disruption, SSEP (SEP) and EEG testing were performed. Additional analysis using FDG-PET was performed approximately 14 days after each sonication. In addition, behavioral scale measurements were obtained continuously throughout the study (Days -7 , $0,7,14,15$, and so forth).

\section{Preparation of Animals}

The study was performed using 2 Papio anubis (olive) baboons (Primates 1 and 2) and 1 Macaca fascicularis (macaque; Primate 3) with weights varying from 8 to $27 \mathrm{~kg}$. The local animal ethics committees approved all animal protocols prior to the start of our experiments. Animals were first anesthetized with an intramuscular injection of a mixture of ketamine $(5 \mathrm{mg} / \mathrm{kg})$ and xylazine $(1 \mathrm{mg} / \mathrm{kg})$. Additional injections were administered if necessary. A peripheral vein was then catheterized for continuous anesthesia with propofol (Fresenius Kabåi); a dose of $0.3 \mathrm{ml} / \mathrm{min}$ was used for device implantation, whereas a lower dose of $0.2 \mathrm{ml} / \mathrm{min}$ was used for sonication sessions. Vital signs were continuously monitored using cardiorespiratory monitoring. When an animal displayed signs of awakening, $1 \mathrm{ml}$ of propofol was immediately administered. Animals were maintained under spontaneous ventilation; however, on signs of respiratory distress, the animals were placed under mechanical ventilation. A unilateral $12-\mathrm{mm}$ bur hole was made on the motor area 7-21 days before the first US treatment, and each animal was implanted with the US device. To localize the motor area of the cortex for device implantation, each animal first underwent presurgery MRI. Then a primate anatomy atlas was used to determine the motor area. The location determined for implantation was measured from the midline laterally and from the supraorbital ridge to place the implant on the cortex motor area. After implanting the device, its location was verified on postsurgery MRI. At the end of the study (4 months after initial implantation), the animals were euthanized by intravenous injection of a lethal dose of pentobarbital (Dolethal $18 \mathrm{mg} / \mathrm{kg}$ ). The brain was then extracted and prepared for histological analysis.

\section{Ultrasound Experimental Setup}

A custom-built, MRI-compatible, implantable, airbacked, 1-MHz single-element transducer was constructed using a 10-mm, flat piezoceramic disc (PZ26, Ferroperm Piezoceramics; Fig. 1). The transducer was operated at a center frequency of $1.05 \mathrm{MHz}$ and a pulse repetition frequency of $1 \mathrm{~Hz}$, with a pulse length of 25,000 cycles (23.2- msec total burst duration). For each session of BBB opening, a bolus of SonoVue contrast agent $(0.1 \mathrm{ml} / \mathrm{kg}$, Bracco Imaging) was injected through a peripheral vein, followed by a flush of $5 \mathrm{ml}$ of saline. The total sonication duration from the end of the saline flush (post-microbubble injection) was maintained at a constant 2 minutes, starting from the end of the microbubble and saline flush, which averaged 11 seconds. Thus, the sonication duration was $131 \pm 9$ seconds (mean \pm standard deviation).

The transducer was mounted in a housing that consisted of implantable-grade PEEK Optima (Invibio Ltd.), and the outer surface of the transducer was covered by a $100-\mu \mathrm{m}-$ thick layer of biocompatible silicon. The transducer was driven using a function generator (HP 33120A, Hewlett Packard) and a radiofrequency amplifier (25 W, Kalmus $125 \mathrm{C} \mathrm{CE}$, Kalmus). The implantable US transducer was connected to the external driving system via a transdermal needle (Fig. 1). The electrical power was monitored using a bidirectional coupler (Mini-Circuits) and a digital oscilloscope (Picoscope 3206B) connected to a laptop computer. A custom software program, written in Python (www. python.org), was used to control the acquisition of voltage signals from the coupler and to calculate the forward and reflected power to the transducer during sonications.

To characterize the acoustic output of the transducer before implantation in each animal, a calibrated hydrophone (HGL-0200, Onda) was used to perform field scans of the pressure distribution at low amplitude. Calibrated hydrophone measurements were performed at a distance of $12 \mathrm{~mm}$ on the acoustic axis at the same levels used during $\mathrm{BBB}$ opening sonications in water and were subsequently de-rated using a factor of $0.5 \mathrm{~dB} / \mathrm{cm}$ to account for attenuation in the brain. This pressure amplitude, hereafter referred to as the "in situ pressure," is used to describe the exposure conditions applied during each sonication. Further details of the calibration procedure are described in our previous work. ${ }^{3}$

\section{Magnetic Resonance Imaging Parameters}

Animals were under general anesthesia during the procedure and immobilized using a custom support system. A 
Philips 1.5-T MR imager with a single-channel birdcage coil was used for the 2 baboons and a Siemens 3-T MR imager with a flexible receiver antenna was used for imaging the macaque. Gadolinium (Gd; Dotarem $0.4 \mathrm{ml} / \mathrm{kg}$ ) was used for contrast-enhanced imaging with T1-weighted sequences to visualize the integrity of the $\mathrm{BBB}$, and additional standard clinical 3D sequences of T2, T2*, FLAIR, and diffusion-weighted imaging (DWI) were performed as well. All MRI sequences were axial with a slice thickness of $3 \mathrm{~mm}$ except for the 3D T1-weighted Gd sequence, which used a slice thickness of $0.8-1.0 \mathrm{~mm}$. All MRI sequences were performed immediately before and after each sonication. Additional MRI was performed at 1,2, and 7 days after some of the sonications. The T1-weighted Gd-enhanced sequence was performed 20 minutes after sonication and 15 minutes after Gd injection. Magnetic resonance imaging results were reviewed using Osirix software (Pixmeo).

The total volume of BBB disruption after US sonications was quantified using the T1-weighted Gd-enhanced image volumes. Each post-sonication acquisition was first rigidly registered to pre-sonication image volumes using 3D Slicer (www.slicer.org). Next, the registered image volumes were imported into ImageJ (imagej.nih.gov/ij/). The background contrast in each image volume was equalized by multiplying the pre-sonication images by a scalar factor based on evaluating the contrast difference in regions of interest (ROIs) outside the US sonication region. Pixelwise division of post- and pre-sonication images was then performed for the 2 image sets, and a binary mask was made by thresholding the images to pixels with only $10 \%$ enhancement or greater. Manual masking of voxels outside the US field in each slice was then performed, as was the removal of voxels that had a low initial signal level. The total number of voxels was then quantified to estimate the total volume of BBB disruption.

\section{Positron Emission Tomography Imaging}

The PET imaging was performed for the 2 baboons on an EXACT HR+ positron tomograph (Siemens) with a spatial resolution at the center of field of view of approximately $5 \mathrm{~mm}$ (note that PET imaging was only possible at the facility where the baboons were treated). The camera has an axial field of view of $15.5 \mathrm{~cm}$, which permits imaging of the brain in one bed position. Repeated imaging protocols of brain glucose metabolism activity using FDG were performed at different time points before the first BBB opening (Day -7) and longitudinally at 14 days after each $\mathrm{BBB}$ disruption (the day prior to the subsequent BBB disruption session) to evaluate long-term potential modification of brain metabolism. For each PET scan, a dynamic series of 34 images was acquired during 60 minutes after measurement of attenuation correction performed on the basis of $68 \mathrm{Ge} / 68 \mathrm{Ga}$ transmission scans. A mean of $8 \pm 0.6$ $\mathrm{Mbq} / \mathrm{kg}$ of FDG was administrated intravenously at the same time as the start of image acquisition. Regions of interest on both hemispheres were drawn on PET images using Anatomist (http://brainvisa.info). Concentrations of radioactivity in the ROIs were calculated for each frame and were expressed as the injected dose per gram of tissue. To normalize the differences in body weight between animals and the variation in injected doses, the standard uptake value (SUV) was calculated at 60 minutes by dividing the "activity in the ROI" by the injected dose per kilogram of body weight. To analyze the total amount of tracer that had accumulated in the different brain hemispheres, the area under the SUV time activity curve (AUC in min*SUV) was also calculated.

\section{Electrophysiology}

Electroencephalography and SSEP monitoring were performed with a NeuronSpectrum device (Neurosoft). Animals were under general anesthesia consisting of propofol the day of sonication and ketamine and xylazine for the control. The EEG and SSEP recordings were performed before and after each sonication, on both hemispheres, with each animal serving as its own control. Additional measurements were performed at Days 1,2, and 7 . Sterile monopolar needles were inserted transcutaneously on the appropriate sites (Spes Medica). Electroencephalography was performed using 8 channels ( 2 frontal, 2 temporal, 2 parietal, 2 occipital, 1 reference channel, and 1 grounded electrode). Each experimental session lasted for 15 minutes. The SSEPs elicited by median nerve and internal popliteal nerve stimulations were studied with the use of 4-channel recordings. Electrical stimulation was delivered via a constant-current stimulator. The stimulation intensity varied between 0 and $13 \mathrm{~mA}$. Reproducibility of stimulation was controlled by the motor response. The intensity of stimulation was adapted from the threshold of adapted movement as a stimulation reference. After determining the appropriate threshold for stimulation, 3 types of stimulations - 1 below the clinical threshold, 1 infralaminar, and 1 for the movement-were delivered. For each nerve SSEP, an average of 200 successive individual responses were recorded. The motor response to assess the stability of stimulation was verified.

\section{Behavioral and Clinical Evaluation}

The goal of the behavioral and clinical evaluation was to detect any clinical signs of neuronal deficit or epileptic signs and to check the general behavior of the animals, in particular, their eating habits. The 3 animals were evaluated twice a day using a motor and behavioral evaluation scale, which was derived from a Parkinson scale for primates. The scale had 10 items: feeding, posture, balance, reactions to stimuli, tremor, moving, posture, motricity of the left and right sides, and eye movement. The final score is the sum of the scores for each item. Possible scores ranged from 0 (normal) to 42 . All primates displayed a normal clinical and behavioral score (0).

\section{Histological Analysis}

After euthanizing each animal, the brain was immediately extracted and cut into $1.5-\mathrm{cm}$ slices, fixed in $4 \%$ paraformaldehyde, and immersed in glucose solutions for 7 days. After fixation, slides were frozen, and tissues were cut into $30-\mu \mathrm{m}$ slices before being stained with $\mathrm{H} \& \mathrm{E}$ (to examine for bleeding or ischemic process), Perls' Prussian blue (for hemosiderin), Bodian (for neuronal-fibrillar degeneration), Fluoro-Jade stain (for neuronal apoptosis), and immunohistochemistry with GLUT1 staining (integ- 


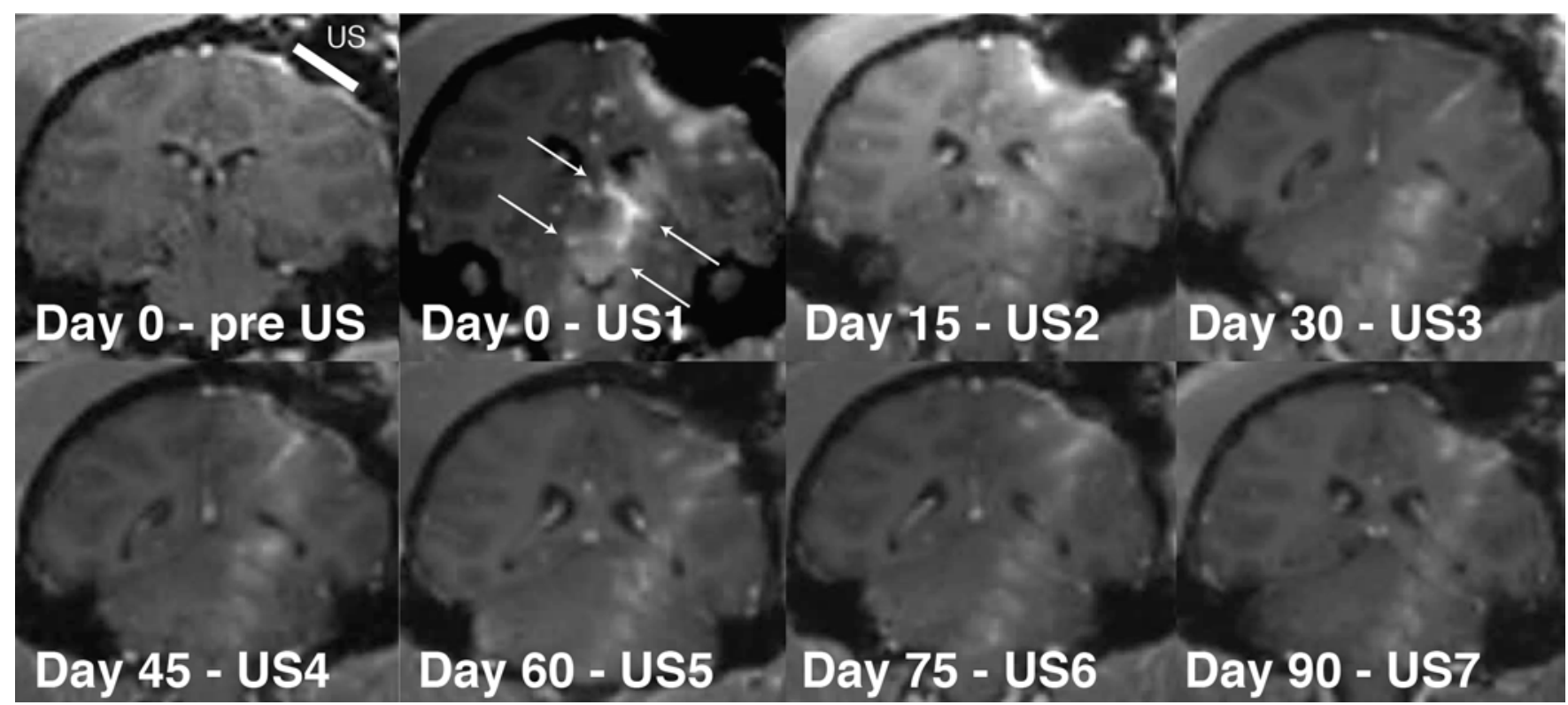

FIG. 3. Disruption of the BBB observed in the macaque (Primate 3) immediately after sonication with the implantable US device. The BBB was opened every 15 days a total of 7 times. Opening of the BBB was demonstrated on T1-weighted contrast-enhanced MR images by a hypersignal in a cylindrical volume in front of the US transducer.

rity of vascular vessel). Light microscopy was performed by an independent neuropathologist (C.A.) who was blinded to the US exposure locations and parameters.

\section{Results}

\section{Magnetic Resonance Imaging Results}

T1-Weighted Contrast Enhancement

All MRI sequences showed normal brain, and no anomalies were observed on any of the imaging sequences prior to device implantation. Each animal underwent MRI with identical sequence parameters approximately 20 minutes before and after each US sonication. Bloodbrain barrier disruption was assessed by localized contrast enhancement and extended to the base of the skull (depth of $3 \mathrm{~cm}$ ), spread in a 1-cm-diameter cylinder. Blood-brain barrier disruption was observed from 0.6 to $0.8 \mathrm{MPa}$ in 6 of 7 sessions in the first baboon, 5 of 7 sessions in the second baboon, and 7 of 7 sessions in the macaque. In 3 of the sessions in the 2 baboons, BBB disruption was not observed after implant activation, an effect that was attributed to technical issues with the device prototype that were corrected by experimentation in the macaque.

Figure 3 shows T1-weighted contrast-enhanced MR images from the macaque for each of the $7 \mathrm{BBB}$ disruption sessions. The BBB disruption observed on the T1-weighted contrast-enhanced images corresponded well with the US field and was observed for all 7 of the sonications.

The total volume of BBB disruption was quantified using T1-weighted contrast-enhanced MRI for the 2 baboons. (Note that the MR images in the macaque were noisier and that the quantitative approach developed herein did not give meaningful values when applied to the macaque data, probably because of the different coil and MR scanner used for the macaque at a different facility.) The volume of BBB disruption was $1.16 \pm 0.71 \mathrm{~cm}^{3}\left(\min =0.46 \mathrm{~cm}^{3}\right.$, $\max$ $=2.35 \mathrm{~cm}^{3}$, results from 9 of 11 sonications). Results of the BBB disruption analysis method for the fifth successful disruption in the first baboon are shown in Fig. 4.

\section{Other MRI Sequences}

After each treatment, a subarachnoid hypersignal was observed on FLAIR images in the sonicated hemisphere, without mass effect on brain parenchyma and without clinical repercussions on awake animals. Figure 5 shows the hypersignal on FLAIR images obtained immediately after and 14 days after BBB disruption. This sequence was also performed with and without $\mathrm{Gd}$ injection to eliminate contrast agent signal. The sequences were similar. No signal anomalies were observed on $\mathrm{T} 2 *$ both immediately after and 14 days after sonication, which ruled out the possibility that the hypersignal observed on FLAIR was caused by hemorrhage. In addition, no image abnormalities were observed on T1, T2, or DWI sequences. The FLAIR hypersignal disappeared when the animals were reimaged the day after sonications.

\section{Behavioral Analysis}

The 3 primates were subject to daily analysis using behavioral and neurological scales. They did not display any modifications in any of their behavioral or neurological signs and did not appear to behave differently, although $\mathrm{BBB}$ disruption was performed on top of the motor cortex. No hemodynamic changes were observed during the treatments. The scores remained at 0 for the 3 animals after the 7 BBB disruption sessions. The animals appeared to be unaffected by repeated BBB disruption. In some sessions, though, a transitory increase (20\%) in the spontaneous re- 




FIG. 4. The volume of BBB disruption was quantified using T1-weighted Gd-enhanced images for each sonication in the baboons (Primates 1 and 2). The region of disruption is shown as a green overlay on T1-weighted images in this example from the fifth BBB disruption in Primate 1, in which the net volume of disruption was $1.54 \mathrm{~cm}^{3}$. Each image is from a separate slice in the MR acquisition with a slice thickness of $0.7 \mathrm{~mm}$. The implant can be observed in the upper left corner of slices 61-70. Great variability in BBB opening was observed after sonications, with a mean value of $1.16 \pm 0.71 \mathrm{~cm}^{3}$ in the 2 baboons $\left(\min =0.46 \mathrm{~cm}^{3}, \max =2.35 \mathrm{~cm}^{3}\right)$.

spiratory frequency was observed during the sonication, with an immediate return to normal respiration by the end of the sonication.

\section{Electrophysiological Results}

Since only 3 animals were used for this study, a group statistical analysis was not performed. Instead, the analyses were performed with each animal serving as its own control since analyses were performed before and after sonication.

\section{Electroencephalography}

Blood-brain barrier rupture may be associated with the apparition of epileptic discharges, and abnormalities of BBB permeability may be involved in epileptogenic processes. ${ }^{11}$ An electroencephalographic exploration was therefore performed before and after sonication to unravel any brain dysfunction or epileptic activities. Control EEG explorations were normal considering the existence of a spatially organized background activity, its amplitude and symmetry, its reactivity, and the absence of abnormal form, including epileptic features. These EEG parameters were monitored before and after sonication. The only variation was a higher amplitude within the sonicated hemisphere, already described during EEG corticography surgery monitoring and suggested to be related to the surgical bone flap. After treatment, no epileptic sign or cerebral slow down, either focal or diffuse, was observed.

\section{Somatosensory Evoked Potential}

Observed peripheral latencies had a low standard deviation and standard error of the mean, showing the reproducibility of the exam. The average amplitude $(\mu \mathrm{V})$ of the response after electrical peripheral stimulation in the different collection points was studied. Typical results are shown in Figs. 6 and 7, which show no EEG abnormalities, conduction blocks, slowing down, or cortico-neuronal sideration.

\section{FDG-PET Glucose Metabolism Imaging Analysis: Primates 1 and 2}

Visual image analysis at the different dates (1 image before the first opening and 6 images after up to 3 months) in Primates 1 and 2 did not reveal any differences (hypo 


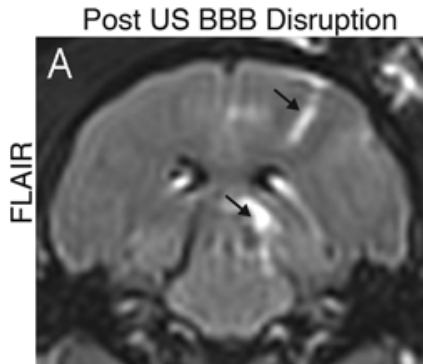

14 days post BBB Disruption


FIG. 5. Hypersignal observed on FLAIR sequences in Primate 3 (macaque) immediately after and 14 days after BBB disruption. The T2* images acquired immediately after and 14 days after sonication did not show any artifacts.

or hyper) in FDG uptake in brain tissues. The mean uptake, expressed in SUVs and shown in Fig. 8 upper, was relatively stable during the period of imaging, with values for each hemisphere ranging from 1.9 to 2.9 with a mean of $2.4 \pm 0.4$ for the male and from 1.5 to 2.3 with a mean of $1.8 \pm 0.2$ for the female. Comparison of right (opening area) and left (control area) hemispheres at 60 minutes by calculation of the SUV ratio provided similar values of
$1.01 \pm 0.01$ for both animals, showing that 14 days after $\mathrm{BBB}$ opening, no differences in glucose metabolism were observed between the 2 hemispheres. An example from Primate 1 is shown in Fig. 8 lower. The measurement of the AUC provided exactly the same value for both sides of the brain $(74 \pm 7$ for male and $62 \pm 7$ for the female in min.SUV) illustrating no differences in FDG uptake. For both baboons, the initial values measured at Day 0 before the first opening provided results similar to those observed later.

\section{Histological Results}

About 180 slices were histologically studied for each animal. Sonicated and nonsonicated hemispheres were compared. Hematoxylin and eosin staining did not indicate any ischemic or bleeding processes. On Perls' staining in a single baboon, mild extravasation of a red cell was observed from a region directly in front of the transducer $(<5 \mathrm{~mm})$. This extravasation, undetectable on T2* MRI sequences, did not have any impact on the neurological status of the primate. Hematoxylin and eosin staining of the dura mater under the transducer indicated hypervascularization, an inflammatory process frequently observed during the postsurgical period and which was probably due to the initial procedure to implant the transducer in the primate (Fig. 9A and B). Fluro-Jade staining did not indicate any signs of neuronal apoptosis in any of the primates (Fig. 9C). No positive Perls' Prussian blue staining was detected. Bodian staining did not display neuronalfibrillar degeneration (Fig. 9D). Immunohistochemistry with GLUT1 antibody showed good integrity of the vascular wall (Fig. 9E).

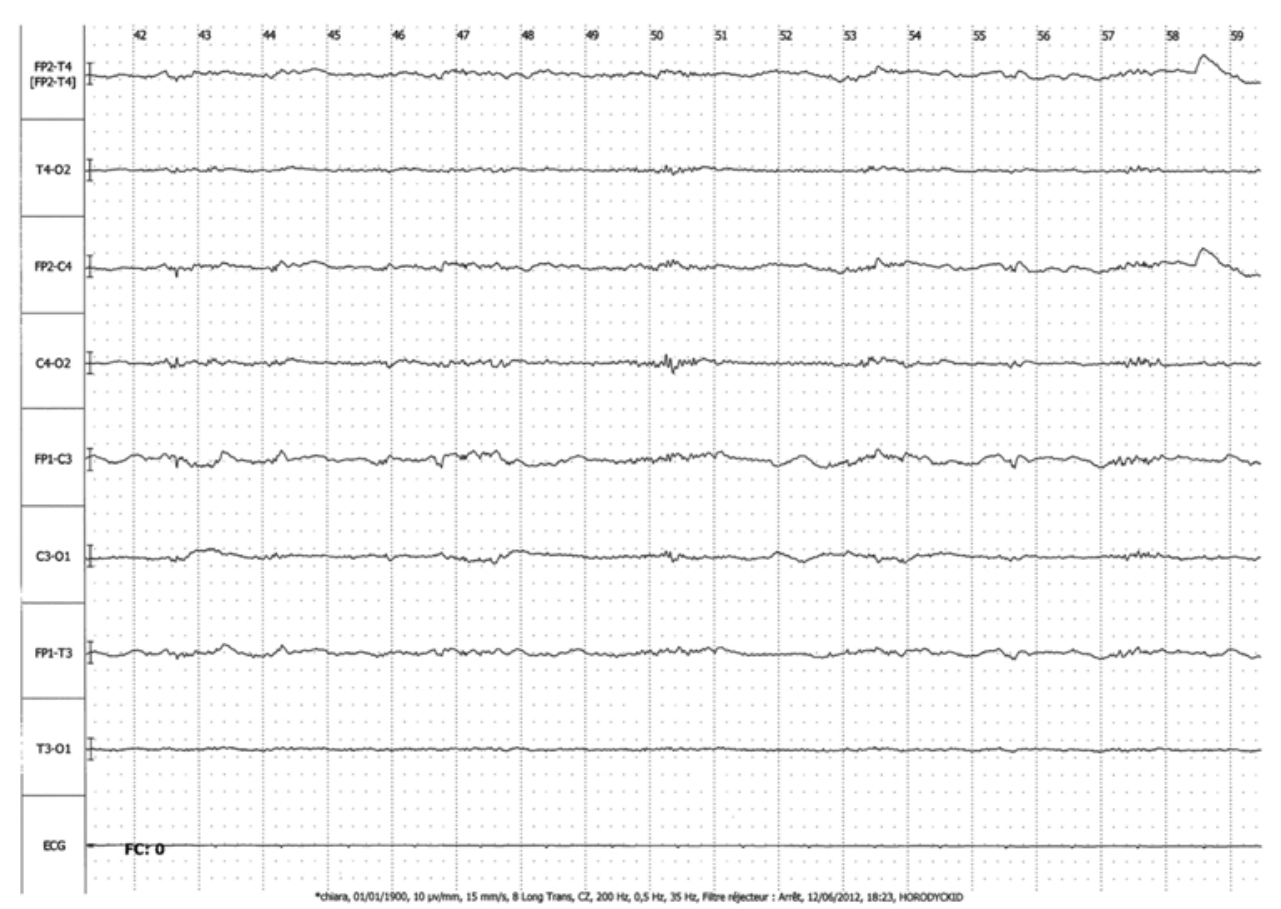

FIG. 6. Electroencephalography tracings postdisruption of the BBB in Primate 2 (baboon). This figure shows no indication of epileptic figures. No differences in the EEG recordings appear before (controls) and after sonication. 

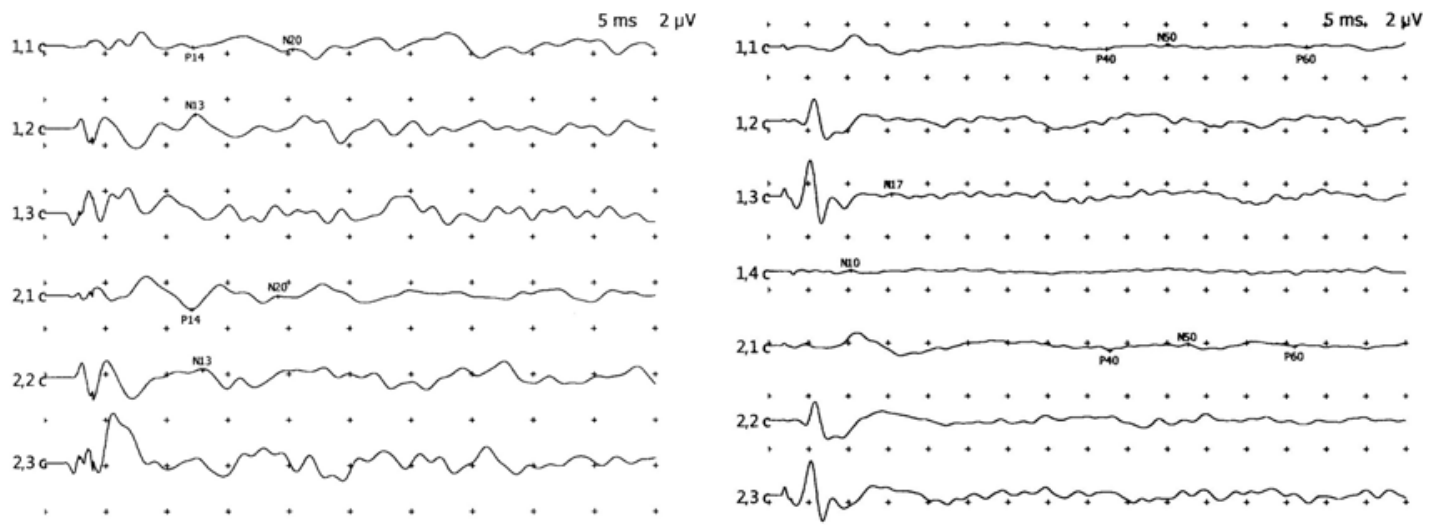

FIG. 7. Somatosensory evoked potentials measured after US disruption of the BBB in Primate 3 (macaque). Left: Median left nerve SSEPs. Right: Popliteal left nerve SSEPs.

\section{Discussion}

The goal of BBB disruption using our implantable US device is to enhance the concentration of drugs that can reach brain tumors, in particular, gliomas, and surrounding infiltrated brain. By enhancing the concentration of drugs that reach (peri)tumoral tissue, the efficacy of intravenous chemotherapy for these types of tumors may be enhanced. In fact, the observed resistance of gliomas to current therapies is attributable to 2 nonexclusive effects: tumor cell resistance (if a chemotherapy is not adapted or is not at a sufficient concentration, the tumor cells may acquire resistance to the drug therapy ${ }^{17,30,31}$ ) and vascular cell resistance (the natural barrier of endothelial cells of the BBB blocks $99 \%$ of all drug therapies, and eventually barrier resistance is acquired for the few drugs that can initially cross, such as small drugs $[<500 \mathrm{D}]$ or new targeted therapies in development).

\section{Efficacy}

In preclinical studies, the enhancement of drug delivery to the brain after BBB disruption with US has been shown to be more than $500 \%$ for drugs that pass the BBB poorly, such as doxorubicin, ${ }^{25}$ and this has been correlated to improved tumor control. For example, tumor growth was slowed in a 9L rat glioma model when BBB opening was combined with the systemic administration of liposomal doxorubicin. ${ }^{25,26}$ Tumor growth was slowed in rats treated with a single US sonication, with tumor doubling rates increasing from 2.7 days in controls to 3.7 days in rats treated with $\mathrm{BBB}$ opening via US. The reduction in the tumor growth rate resulted in a $24 \%$ enhancement in median survival. ${ }^{26}$

Aryal et al. ${ }^{2}$ performed studies in a 9L rat glioma model but used multiple US treatments combined with the systemic administration of liposomal doxorubicin (Doxil/ Caelyx). Three treatments were performed over the course of 3 weeks ( 1 treatment per week). Median survival in the treated rats was increased by $100 \%$ as compared with that in controls. Furthermore, $40 \%$ of the treated animals survived for more than 140 days, whereas all control rats died after less than 30 days. These preclinical results show that there is strong interest in disrupting the BBB to increase the concentration of chemotherapeutics in gliomas as survival can be significantly enhanced.

\section{Device Design}

The design of the implantable US transducer tested in our studies was defined by practical clinical needs. An unfocused US beam was chosen as it is better suited to treating patients with diffuse pathologies such as gliomas. An US system implantable in the skull thickness through a bur hole was designed to avoid the complications of focusing US energy through the skull bone and to eliminate the need for MRI or CT correction methods.

Since the device is implanted, it is designed to be MRcompatible to permit MRI follow-up of tumors and to be easily and safely used in routine clinical practice during multiple chemotherapy sessions. The device developed herein is implanted through a bur hole during either biopsy or resection for histopathological diagnosis, and the skin is closed as usual, covering the device. At each further chemotherapy session, the device is connected to an external radiofrequency generator for 2 minutes by using a transcutaneous bipolar needle connection. After 2 minutes of sonication, the needle is removed and the BBB remains open for a period of 6 hours or more, although the magnitude of the opening begins to decrease immediately following the initial disruption. ${ }^{22}$

The T1-weighted contrast enhancement after Gd injection permitted us to confirm BBB opening for each sonication session. The volume of BBB opening quantified from the Gd-enhanced T1 images was between 0.46 and $2.35 \mathrm{~cm}^{3}$. These volumes corresponded well with the initial estimate that $\mathrm{BBB}$ disruption corresponds to a 5- to $10-\mathrm{mm}$-diameter cylindrical volume that extends from the 1-cm-diameter transducer face to the skull base, a distance of approximately $3 \mathrm{~cm}$ in the primate brain.

The wide variability observed in the BBB volume disruption estimate across the US treatments may have been attributable to 2 different effects. The first possibility is an actual difference that influenced the overall volume of the BBB disruption that was performed. Such effects may have included the preparation and injection of the microbubble solution, the influence of anesthesia on cerebral blood flow during sonications, or the influence of endo- 

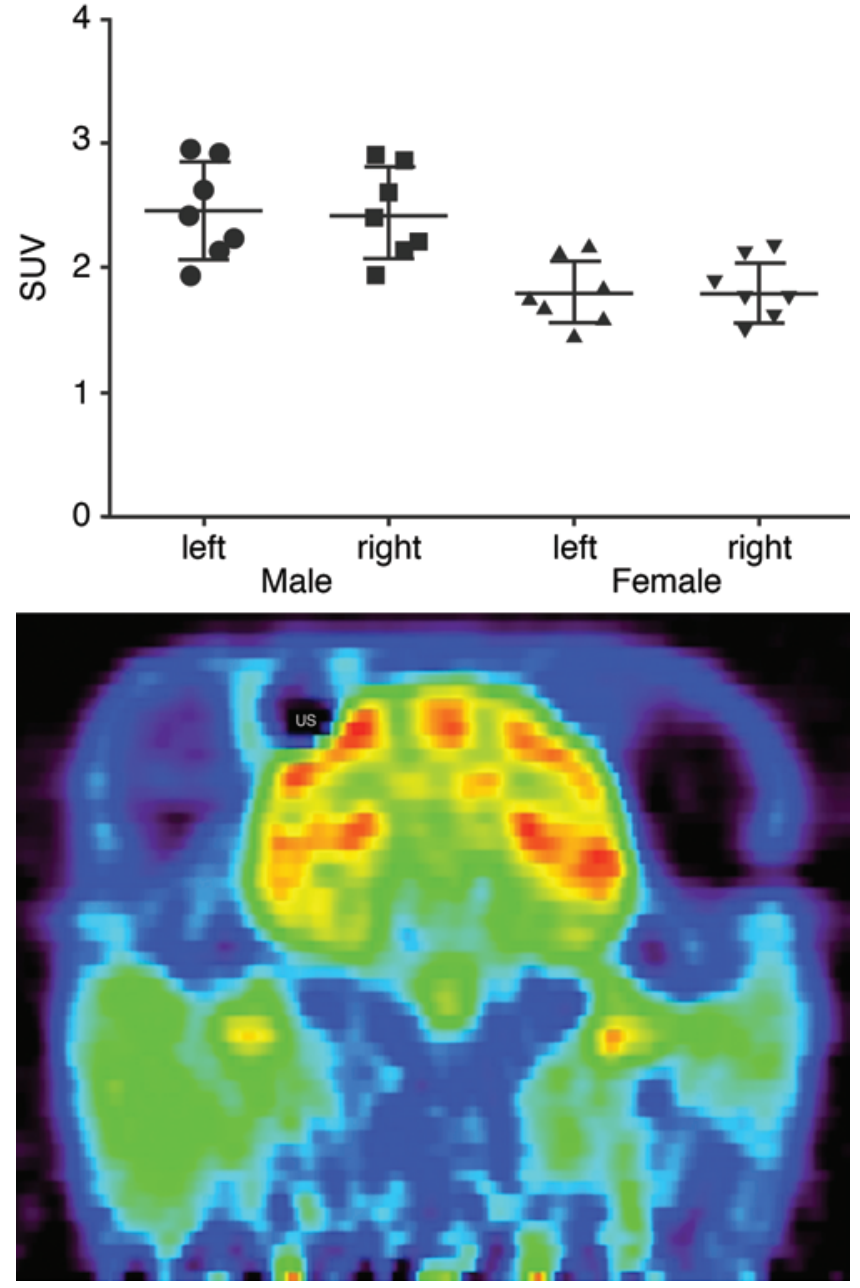

FIG. 8. Upper: FDG quantification in the left (control) and right (sonicated/BBB opening) hemispheres in male and female primates, expressed as SUVs. Each individual measurement corresponded to a dot, square, upright triangle, or upside down triangle. The mean values of both sides were not significantly different (Wilcoxon test); thus, no changes in glucose uptake were observed 14 days after each BBB opening session. Lower: FDG visual image from Primate 1 (baboon) at 11 days after the fifth sonication to disrupt the BBB. The implanted US transducer can be seen in the upper left corner. No changes were observed in glucose uptake during the course of the study.

thelial vascular spasm, which has been observed in other studies. ${ }^{12,23}$

The second possibility to explain the variability relates to the processing method developed, which was somewhat empirical. An absolute quantitative method such as an estimate of the vessel permeability using dynamic contractenhanced (DCE) imaging may be better suited to such an analysis and will be explored in future studies. ${ }^{22}$

Although a mean volume of only $1.16 \mathrm{~cm}^{3}$ was calculated as the zone of BBB disruption, the actual zone of enhanced drug delivery is larger. Here, a $10 \%$ enhancement in contrast was used for signal-to-noise considerations in the processing method, even though the BBB was disrupted beyond this region. In addition, in a similar study in which carboplatin, a chemotherapy drug that does not normally cross the $\mathrm{BBB}$, was quantified after $\mathrm{BBB}$ disruption in a primate model, we found drug concentrations were increased by $30 \%$, even at $10 \mathrm{~mm}$ from the US beam axis. ${ }^{9}$ The analysis performed in the present study was based solely on the distribution of a bolus injection of $\mathrm{Gd} 20$ minutes after sonications. Future studies are needed to examine the correlation between this metric and that of absolute drug concentrations measured in the brain after BBB disruption.

\section{Safety}

Our results in large-animal models demonstrate that US-induced BBB disruption is safe, even with repeated disruption of the BBB every 15 days. These results agree well with the recent work of McDannold et al., ${ }^{19}$ who used a clinical extracranial focused US system operating at 220 $\mathrm{kHz}$ to repeatedly open the $\mathrm{BBB}$ in an approximately $1-\mathrm{cm}^{3}$ zone in a target in the central visual field in primates. A safe acoustic pressure level for BBB opening was determined, and repeated opening of the $\mathrm{BBB}$ was performed up to 13 times over 26 weeks. Repeated BBB opening was found to be safe, with no behavioral or visual deficits or loss in visual acuity. Similar results validating the long-term safety of $\mathrm{BBB}$ disruption in primate models with a focused US system were also observed in the recent work of Downs et al. ${ }^{7}$

In our study, MRI analysis did not indicate any bleeding, ischemic, or necrotic processes. Neither were corresponding effects observed on histological analysis of these same regions. The T1-weighted Gd MRI sequence performed at Day 1 post-sonication showed no contrast enhancement and the disappearance of FLAIR hypersignal, proving that $\mathrm{BBB}$ disruption is reversible. Furthermore, no hypointense regions were observed at 15 days after sonications (just prior to the subsequent treatment). Hynynen et al. ${ }^{14}$ observed similar effects in a rabbit model and found that while some damage to capillaries was observed at pressures used to disrupt the BBB (0.5 MPa), minor damage to capillaries was not associated with damage to neurons.

The small number of animals included in this study due to ethical considerations was compensated by recurrent $\mathrm{BBB}$ disruption $(\times 7)$ and repetitive multimodality exams to demonstrate the safety of BBB disruption on brain parenchyma. The analyses of morphological (absence of bleeding, necrosis, ischemia), metabolic (looking for modification of brain metabolism), and functional (looking for epileptic signs, conduction troubles, behavior troubles, and so forth) measures did not show any signs of toxicity for the $21 \mathrm{BBB}$ disruption sessions.

Electrophysiology studies during the 3 months did not display any epileptic signs or conduction issues. However, the study was limited as general anesthesia was used during exams. These exams could not be performed in awake conditions given the difficulty in performing such studies in awake primates as well as the movement artifacts. Propofol can hide some epileptic forms, but no epileptic symptoms were observed when animals were awake either. Variability among the 3 animals because of species, sex, and age compromises comparison but still offers safety data on such $\mathrm{BBB}$ disruption processes in a heterogeneous population.

\section{Further Development}

Considering the safety data obtained in this experiment 

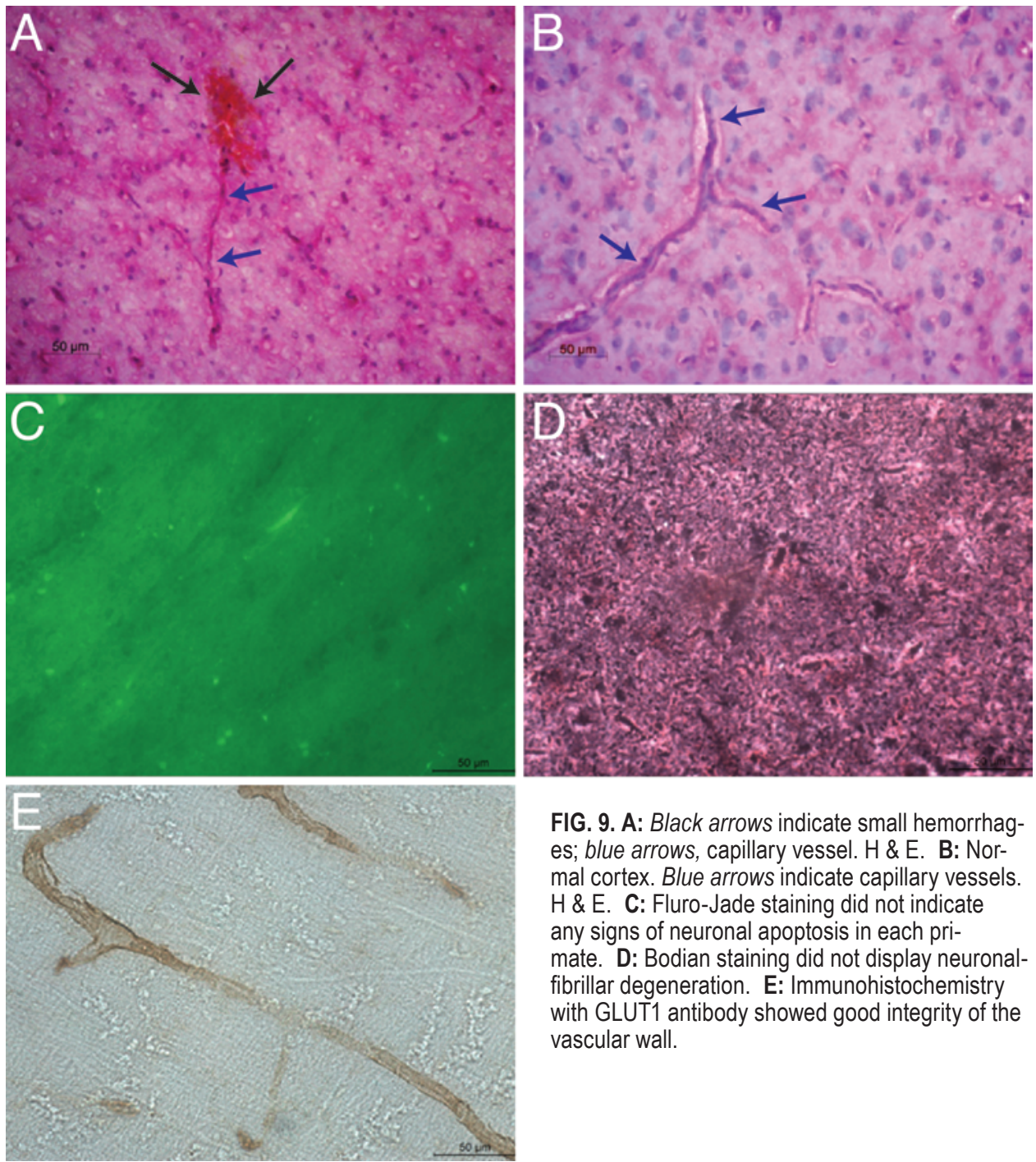

FIG. 9. A: Black arrows indicate small hemorrhages; blue arrows, capillary vessel. H \& E. B: Normal cortex. Blue arrows indicate capillary vessels. $\mathrm{H} \& \mathrm{E}$. C: Fluro-Jade staining did not indicate any signs of neuronal apoptosis in each primate. D: Bodian staining did not display neuronalfibrillar degeneration. E: Immunohistochemistry with GLUT1 antibody showed good integrity of the vascular wall.

and previous efficacy studies, ${ }^{3,4}$ a Phase I/IIa clinical trial in patients is envisioned for the near future.

\section{Conclusions}

Results of this study demonstrate that an implantable, 1-MHz unfocused US transducer can be used to safely and repeatedly open the BBB. Such an approach may be used to enhance the efficacy of chemotherapy or other drug treatments for brain disorders.

\section{Acknowledgments}

We gratefully acknowledge Alejandra Uzcategui Pedroza for help with programming the computer interface used for controlling treatments; Pascal Merlet, Raphael Boisgard, and Rose-Marie Dubuisson for access to CEA Orsay; Mathieu Santin and Annick Prigent for access to ICM; and Guillaume Bouchoux for useful feedback on the image analysis methods.

\section{References}

1. Abbott NJ, Romero IA: Transporting therapeutics across the blood-brain barrier. Mol Med Today 2:106-113, 1996

2. Aryal M, Vykhodtseva N, Zhang YZ, Park J, McDannold N: Multiple treatments with liposomal doxorubicin and ultrasound-induced disruption of blood-tumor and blood-brain barriers improve outcomes in a rat glioma model. J Control Release 169:103-111, 2013

3. Beccaria K, Canney M, Goldwirt L, Fernandez C, Adam $\mathrm{C}$, Piquet J, et al: Opening of the blood-brain barrier with an unfocused ultrasound device in rabbits. J Neurosurg 119:887-898, 2013

4. Beccaria K, Canney M, Goldwirt L, Fernandez C, Piquet J, Perier MC, et al: Ultrasound-induced opening of the blood-brain barrier to enhance temozolomide and irinotecan delivery: an experimental study in rabbits. J Neurosurg [Epub ahead of print November 13, 2015. DOI: 10.3171/2015.4.JNS14289]

5. Donelli MG, Zucchetti M, D'Incalci M: Do anticancer agents reach the tumor target in the human brain? Cancer Chemother Pharmacol 30:251-260, 1992

6. Doolittle ND, Miner ME, Hall WA, Siegal T, Jerome E, Osztie E, et al: Safety and efficacy of a multicenter study using intraarterial chemotherapy in conjunction with osmotic opening of the blood-brain barrier for the treatment of patients with malignant brain tumors. Cancer 88:637-647, 2000

7. Downs ME, Buch A, Sierra C, Karakatsani ME, Teichert T, 
Chen S, et al: Long-term safety of repeated blood-brain barrier opening via focused ultrasound with microbubbles in non-human primates performing a cognitive task. PLoS One 10:e0125911, 2015 (Erratum in PLoS One 10:e0130860, 2015)

8. Drappatz J, Brenner A, Wong ET, Eichler A, Schiff D, Groves MD, et al: Phase I study of GRN1005 in recurrent malignant glioma. Clin Cancer Res 19:1567-1576, 2013

9. Goldwirt L, Canney M, Horodyckid C, Poupon J, Mourah S, Vignot A, et al: Enhanced brain distribution of carboplatin in a primate model after blood-brain barrier disruption using in implantable ultrasound device. Cancer Chemother Pharmacol 77:211-216, 2015

10. Groothuis DR: The blood-brain and blood-tumor barriers: a review of strategies for increasing drug delivery. Neuro Oncol 2:45-59, 2000

11. Heinemann U, Kaufer D, Friedman A: Blood-brain barrier dysfunction, TGF $\beta$ signaling, and astrocyte dysfunction in epilepsy. Glia 60:1251-1257, 2012

12. Hynynen K, Chung AH, Colucci V, Jolesz FA: Potential adverse effects of high-intensity focused ultrasound exposure on blood vessels in vivo. Ultrasound Med Biol 22:193-201, 1996

13. Hynynen K, McDannold N, Martin H, Jolesz FA, Vykhodtseva N: The threshold for brain damage in rabbits induced by bursts of ultrasound in the presence of an ultrasound contrast agent (Optison). Ultrasound Med Biol 29:473-481, 2003

14. Hynynen K, McDannold N, Sheikov NA, Jolesz FA, Vykhodtseva N: Local and reversible blood-brain barrier disruption by noninvasive focused ultrasound at frequencies suitable for trans-skull sonications. Neuroimage 24:12-20, 2005

15. Hynynen K, McDannold N, Vykhodtseva N, Jolesz FA: Noninvasive MR imaging-guided focal opening of the bloodbrain barrier in rabbits. Radiology 220:640-646, 2001

16. Hynynen K, McDannold N, Vykhodtseva N, Raymond S, Weissleder R, Jolesz FA, et al: Focal disruption of the bloodbrain barrier due to $260-\mathrm{kHz}$ ultrasound bursts: a method for molecular imaging and targeted drug delivery. J Neurosurg 105:445-454, 2006

17. Jiang P, Mukthavaram R, Chao Y, Bharati IS, Fogal V, Pastorino $S$, et al: Novel anti-glioblastoma agents and therapeutic combinations identified from a collection of FDA approved drugs. J Transl Med 12:13, 2014 (Erratum in J Transl Med 12:126, 2014)

18. Marquet F, Tung YS, Teichert T, Ferrera VP, Konofagou EE: Noninvasive, transient and selective blood-brain barrier opening in non-human primates in vivo. PLoS One 6:e22598, 2011

19. McDannold N, Arvanitis CD, Vykhodtseva N, Livingstone MS: Temporary disruption of the blood-brain barrier by use of ultrasound and microbubbles: safety and efficacy evaluation in rhesus macaques. Cancer Res 72:3652-3663, 2012

20. McDannold N, Vykhodtseva N, Hynynen K: Blood-brain barrier disruption induced by focused ultrasound and circulating preformed microbubbles appears to be characterized by the mechanical index. Ultrasound Med Biol 34:834-840, 2008

21. O'Reilly MA, Huang Y, Hynynen K: The impact of standing wave effects on transcranial focused ultrasound disruption of the blood-brain barrier in a rat model. Phys Med Biol 55:5251-5267, 2010

22. Park J, Zhang Y, Vykhodtseva N, Jolesz FA, McDannold NJ: The kinetics of blood brain barrier permeability and targeted doxorubicin delivery into brain induced by focused ultrasound. J Control Release 162:134-142, 2012

23. Raymond SB, Skoch J, Hynynen K, Bacskai BJ: Multiphoton imaging of ultrasound/Optison mediated cerebrovascular effects in vivo. J Cereb Blood Flow Metab 27:393-403, 2007

24. Santin MD, Debeir T, Bridal SL, Rooney T, Dhenain M: Fast in vivo imaging of amyloid plaques using $\mu$-MRI Gd-staining combined with ultrasound-induced blood-brain barrier opening. Neuroimage 79:288-294, 2013
25. Treat LH, McDannold N, Vykhodtseva N, Zhang Y, Tam K, Hynynen K: Targeted delivery of doxorubicin to the rat brain at therapeutic levels using MRI-guided focused ultrasound. Int J Cancer 121:901-907, 2007

26. Treat LH, McDannold N, Zhang Y, Vykhodtseva N, Hynynen $\mathrm{K}$ : Improved anti-tumor effect of liposomal doxorubicin after targeted blood-brain barrier disruption by MRI-guided focused ultrasound in rat glioma. Ultrasound Med Biol 38:1716-1725, 2012

27. Vykhodtseva N, McDannold N, Hynynen K: Progress and problems in the application of focused ultrasound for bloodbrain barrier disruption. Ultrasonics 48:279-296, 2008

28. Wang F, Cheng Y, Mei J, Song Y, Yang YQ, Liu Y, et al: Focused ultrasound microbubble destruction-mediated changes in blood-brain barrier permeability assessed by contrastenhanced magnetic resonance imaging. J Ultrasound Med 28:1501-1509, 2009

29. White E, Bienemann A, Taylor H, Hopkins K, Cameron A, Gill $\mathrm{S}$ : A phase I trial of carboplatin administered by convectionenhanced delivery to patients with recurrent/progressive glioblastoma multiforme. Contemp Clin Trials 33:320-331, 2012

30. Whittle IR, Malcolm G, Jodrell DI, Reid M: Platinum distribution in malignant glioma following intraoperative intravenous infusion of carboplatin. Br J Neurosurg 13:132-137, 1999

31. Wolff JE, Trilling T, Mölenkamp G, Egeler RM, Jürgens H: Chemosensitivity of glioma cells in vitro: a meta analysis. J Cancer Res Clin Oncol 125:481-486, 1999

\section{Disclosures}

This work was supported by CarThera SAS. Drs. Carpentier, Lafon, Chapelon, and Canney have ownership interest in CarThera SAS. Dr. Horodyckid and Mr. Vignot are employees of CarThera SAS. Drs. Carpentier, Lafon, Chapelon, and Canney have submitted a patent application related to this work. Drs. Carpentier and Canney are consultants for CarThera.

\section{Author Contributions}

Conception and design: Carpentier, Canney, Lafon, Chapelon. Acquisition of data: Carpentier, Horodyckid, Canney, Vignot, Boisgard, Prigent, Santin. Analysis and interpretation of data: Carpentier, Horodyckid, Canney, Boisgard, Drier, Huberfeld, Francois, Prigent, Adam, Willer. Drafting the article: Carpentier, Horodyckid, Canney. Critically revising the article: Carpentier, Horodyckid, Canney. Reviewed submitted version of manuscript: Carpentier, Horodyckid, Canney. Approved the final version of the manuscript on behalf of all authors: Carpentier. Administrative/technical/material support: Lafon. Study supervision: Lafon, Chapelon.

\section{Supplemental Information}

\section{Previous Presentations}

Portions of this work were presented as an oral presentation at the International Symposium on Therapeutic Ultrasound held in Shanghai, People's Republic of China, on May 12-15, 2013, and at the IEEE-UFFC International Ultrasonics Symposium held in Prague, Czech Republic, on July 21-25, 2013; in poster form at the 3rd Symposium on Current and Future Applications of Focused Ultrasound held in Bethesda, Maryland, on October 14-17, 2012; and as an oral presentation at the 4th Symposium on Current and Future Applications of Focused Ultrasound held in Bethesda, Maryland, on October 12-16, 2014.

\section{Correspondence}

Alexandre Carpentier, Department of Neurosurgery, Pitie-Salpetriere Hospital, Assistance Publique Hôpitaux de Paris, Sorbonne University, Paris 6 School of Medicine, Paris 75013, France. email: alexandre.carpentier@aphp.fr. 